\title{
Environmentally dependent bond-order potentials: New developments and applications
}

\author{
D NGUYEN-MANH*, D G PETTIFOR, D J H COCKAYNE, M MROVEC ${ }^{\dagger}$, \\ S ZNAM ZN $^{\dagger}$ and V VITEK \\ Department of Materials, University of Oxford, Parks Road, Oxford OX1 3PH, UK \\ 'Department of Materials Science and Engineering, University of Pennsylvania, Philadelphia, \\ Pennsylvania 19104-6272, USA
}

\begin{abstract}
The bond-order potentials (BOPs) idea employs the orthogonal two-centre tight-binding (TB) representation for the bond energy and the Harris-Foulkes approximation for the repulsive pairwise contribution. In the last ten years, although many efforts have been focused on theoretical calculations of the bond order expression, the BOPs still suffers from the uncertainty of how best to choose the semi-empirical TB parameters that enter the scheme. In this paper, we review recent developments to obtain the reliable and transferable BOPs which help to extend the accuracy and applicability to technologically important multicomponent systems. Firstly, we have found that a simple pair potential is unsuitable for describing the environmental screening effects due to the $s$ and $p$ orbital overlap repulsion in transition metal alloys and therefore the inability to reproduce the negative Cauchy pressures exhibiting in strong covalent systems. By adding the environmental dependent repulsive term, the Cauchy pressure problem has been removed and we are now able to get the BOPs for studying dislocations, extended defects and mechanical properties of hightemperature intermetallic $\mathbf{~ T i - A l ~ a l l o y s . ~ I n ~ p a r t i c u l a r , ~ n e w ~ r e s u l t s ~ o n ~ t h e ~ c o r e ~ s t r u c t u r e s ~ a n d ~ p o s s i b l e ~ d i s s o c i a - ~}$ tion of different type of dislocations will be discussed. Secondly, we present the first derivation of explicit analytic expressions for environmental dependence of $\sigma, \pi$ and $\delta$ bond integrals by inverting the non-orthogonal matrix. We illustrate the power of this new formalism by showing that it not only captures the transferability of bond integrals between $\mathrm{Mo}, \mathrm{Si}$ and $\mathrm{MoSi}_{2}$ but also predicts the large discontinuities between first and second nearest neighbours for $p p \sigma, p p \pi$ and $d d \pi$ even though absence of any discontinuity for the $d d \sigma$ bond integral. A new environmentally dependent BOPs has been developed for $b c c$-Mo indicating that the core structure of $1 / 2\langle 111\rangle$ screw dislocations is narrower than structures found in previous studies in agreement with recent $a b$ initio calculations. Finally, the new formalism will allow us to study the problem of medium range order found recently in amorphous materials with covalent bonding at large and realistic nanoscale. For the case of $a-\mathrm{C}$ where the issue of $s p^{2}$ vs $s p^{3}$ is very crucial for modelling amorphous structure we found that the $\sigma$ and $\pi$ bond integrals are not only transferable between graphite and diamond structures but they are also strongly anisotropic due to inter-plan bonding between graphite sheets.
\end{abstract}

Keywords. Bond-order potentials; environmental dependence; transferability; intermetallic alloys; carbon materials.

\section{Introduction}

The modelling and simulation of materials in general and of intermetallic alloys in particular is a challenging task as it often involves linking the world of the electron theorist to the world of the continuum engineer, thereby spanning at least twelve orders of magnitude in either length or time (Nguyen-Manh et al 1995). This linking is achieved by successively coarse-graining the problem (Pettifor 1997). Firstly, the electronic degrees of freedom may be removed by imagining the atoms to be held together by some sort of glue or interatomic potential, thereby allowing large scale atomistic simulations to be performed on fracture at crack tips, for example. Secondly,

\footnotetext{
*Author for correspondence
}

the atoms themselves may be coarse-grained by grouping them together into cells which interact via deterministic or stochastic rules, thereby allowing, for example, the simulation of microstructure evolution during the growth of single crystal turbine blades. Finally, the continuum world may be reached by averaging over the microstructure and describing the materials through a set of constitutive relationships.

In this paper we outline the new development of environmentally dependent bond-order potentials that are obtained directly by bridging the electronic to atomistic modelling hierarchies with a tight-binding (TB) framework. Figure 1 shows the modelling hierarchy of bridging the gap between electronic and atomistic levels. We will present, in $\$ 2$, the need for reliable angularly dependent interatomic potentials and reassert recent developments of conventional bond-order potentials (BOPs), so 


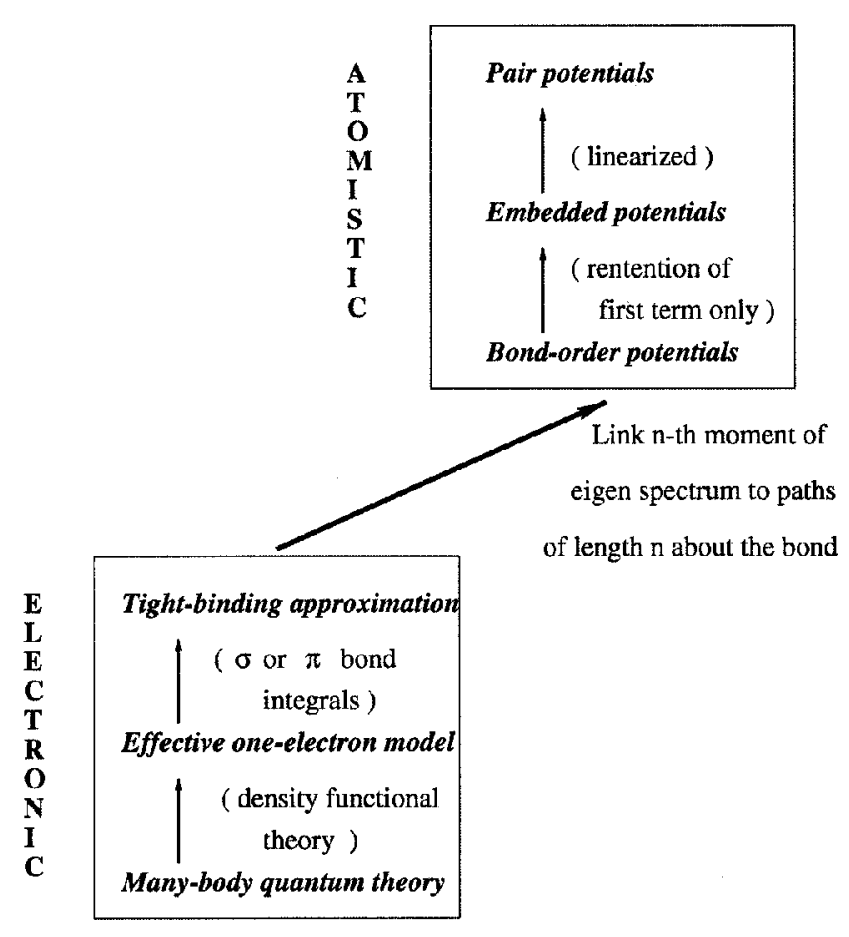

Figure 1. Bridging the gap between electronic and atomistic hierarchies.

that they provide the first 'classical' interatomic potentials that handle both structural differentiation and radical formation naturally.

Unfortunately, BOPs which were based on conventional TB parameters, were not able to satisfy the negative Cauchy pressures for many strongly covalent bonded materials, viz. that $C_{12}-C_{44}<0$ for cubic materials, or $C_{13}-C_{44}<0$ and $C_{12}-C_{66}<0$ for tetragonal or hexagonal ones. The failure of BOPs to yield negative Cauchy pressures resides in the fact that the orbitals used in the conventional two-centre TB approximation are not screened by their local environment (Nguyen-Manh et al 1998). In §3, we will discuss how the environmental dependence will be included within BOPs formalism for producing negative Cauchy, for example, in the case of titanium aluminides which is one of the most promising high-temperature intermetallics alloys with many attractive specific properties for aerospace applications (Dimiduk 1999). In \$4, we show that it is possible to derive an analytic expression for the environmental dependence for the two-centre TB bond integrals starting from the nonorthogonal representation and using recently developed bounded BOPs theory (Nguyen-Manh et al 2000; Pettifor and Oleinik 2000). We will illustrate the technique by considering the transferability of bond integrals within $b c c$-like materials between $b c c$ Mo and binary $\mathrm{MoSi}_{2}$, the latter being an important next generation high-temperature intermetallics (Vasudevan et al 1999). The problem of anisotropy of interaction, for example, in graphite, will be discussed within our new environmentally dependent scheme in $\S 5$ and the conclusion will be gi

\section{Conventional BOPs formalism}

Within the two-centre orthogonal tight-binding bond model (Sutton et al 1988), the binding energy can be written in the form

$$
U=U_{\text {rep }}^{\text {pair }}+U_{\text {bond }}
$$

where $U_{\text {rep }}^{\text {pair }}$ is an empirical repulsive pairwise contribution and the attractive bond energy is given by

$$
U_{\text {bond }}=\frac{1}{2} \sum_{i \neq j} \sum_{j}\left[2 \beta\left(R_{i j}\right) \Theta_{i j}\right],
$$

where $\beta\left(R_{i j}\right)$ is the bond integral between a given pair of atoms $i$ and $j$, a distance $R_{i j}$ apart and $\Theta_{i j}$ is the bond order. Equation (2) has been written following Coulson's (1939) idea that the global description of energy bands with an associated band energy could be broken down into a local description of covalent bonds with associated individual bond energies. The bond order, which is onehalf the difference between the number of electrons in the bonding state compared to the anti-bonding state, can be expressed in term of the intersite Green's function matrix element, $G_{i j}(\varepsilon)$ as

$$
\Theta_{i j}=-\frac{2}{\pi} \operatorname{Im} \int^{\varepsilon_{\mathrm{F}}} G_{i j}(\varepsilon) \mathrm{d} \varepsilon,
$$

where $G_{i j}(\varepsilon)=\left\langle i\left|(\varepsilon-\hat{H})^{-1}\right| j\right\rangle$ and $\hat{H}$ the TB Hamiltonian operator. Thus, the chemists' bond order, $\Theta_{i j}$, is none other than twice the physicists' off-diagonal density matrix element which is implicit in (3). As it is shown from figure 1, a link from TB eigenspectrum at the electronic level to BOPs is coarse-grained through its moments, viz. the $p$ th moment is related to the sum over all self-returning bonding or hopping paths of length $p$ as

$$
\begin{aligned}
\mu_{p} & =\frac{1}{N} \sum_{n} \varepsilon_{n}^{p}=\frac{1}{N} \sum_{n}\left(H^{p}\right)_{n n}=\frac{1}{N} \operatorname{Tr} H^{p} \\
& =\frac{1}{N} \sum_{i_{1}, i_{2} \ldots i_{p}} H_{i_{1}, i_{2}} H_{i_{2}, i_{3}} \ldots H_{i_{p}, i_{1}} .
\end{aligned}
$$

Hence, the second moment or mean square width of the spectrum is obtained from self-returning paths of length two, whereas the fourth moment which measures the unimodal versus bimodal behaviour is obtained from selfreturning paths of length four (Pettifor et al 1995). As is well documented, expansions of functions in terms of their moments are usually very poorly behaved and 
therefore the diagonal element of a Green's function, $G_{00}(\varepsilon)$ can be better expressed as a continued fraction by using the Lanczos recursion algorithm. This transforms the sparse TB Hamiltonian with respect to the atomic orbitals $|i\rangle$ into tri-diagonal form with respect to the Lanczos basis $\left|u_{n}\right\rangle$ with corresponding matrix elements, $a_{n}=\left\langle u_{n}|\hat{H}| u_{n}\right\rangle$ and $b_{n}=\left\langle u_{n-1}|\hat{H}| u_{n}\right\rangle$. The BOPs theory relies on a novel theorem (Aoki 1993) that expresses the off-diagonal Green's function matrix elements, $G_{i j}$, as the derivative of a diagonal element, viz.

$$
G_{i j}(\varepsilon)=\frac{\partial}{\partial \lambda} G_{00}^{\lambda}(\varepsilon)
$$

Very recently, the convergence of off-diagonal Green's function matrix elements has been speeded-up dramatically by constraining the poles of $G_{i j}$ to be the same as those of the average on-site Green's function $(1 / 2)\left(G_{i i}+G_{j j}\right) \quad$ (Pettifor and Oleinik 2000). This has allowed the derivation of explicit analytic expressions for $\sigma$ and $\pi$ bond orders that reproduce accurately the TB results from direct matrix diagonalization. The $\sigma$ bond order, for example, can be written as

$$
\Theta_{i j, \sigma}=\frac{1}{\sqrt{1+\frac{2 \Phi_{2 \sigma}+\hat{\delta}^{2}}{1+\sqrt{\frac{\Phi_{4 \sigma}-2 \Phi_{2 \sigma}^{2}+\Phi_{2 \sigma}^{i} \Phi_{2 \sigma}^{j}}{\Phi_{2 \sigma}}}}}},
$$

where $\Phi_{n \sigma}=(1 / 2)\left(\Phi_{n \sigma}^{i}+\Phi_{n \sigma}^{i}\right)$. The two-hop, $\Phi_{2 \sigma}^{i}$, and four-hop, $\Phi_{4 \sigma}^{i}$, contributions that start and end on atom $i$ with explicit angular dependence as well as the atomic energy level separation, $\hat{\delta}$, in (6) are given in Pettifor and Oleinik (2000). An analytic expression for the $\pi$ bond in terms of neighbouring bond angles and dihedral angles may also be derived. The widely used Tersoff (1986) potential is analogous to retaining only the second-moment contribution in (6) so that the bond order varies as $\left(1+\hat{\delta}^{2}+2 \Phi_{2 \sigma}\right)^{-1 / 2}$. However, the predicted values of Tersoff-type bond order provide an accurate measurement of the $\sigma$ bond order that would lead to a big error in the $\sigma$ bond energy, $2 \beta_{\sigma} \Theta_{\sigma}$. These large errors are because the small energy differences in binding energy between different non-close-packed structure types are driven by the fourth moment paths of length four that determine the unimodal versus bimodal shape of the electronic spectrum rather than the paths to length two that determine the mean square width of the spectrum. Including the BOPs predictions for the $\pi$ bond orders can help to reproduce the bond energy not only for the single, double and triple but also for the conjugate bonds in benzene and graphite.

The above derivation of BOPs formalism depends critically on the availability of reliable orthogonal TB parameters. During the past few years it has become recognized that robust schemes require both pairwise and bond contributions from (1) to be environmentally dependent (Tang et al 1996). Below we will discuss why it is crucial to include this idea into the BOPs and how it is implemented in constructions of the BOPs in realistic modelling of materials.

\section{Environmentally dependent effect, $I$ : screening of atoms}

\subsection{Negative Cauchy pressure problem}

It is well known that the embedded atom method (Daw and Baskes 1984) or Finnis-Sinclair (Finnis and Sinclair 1984) potentials predict positive Cauchy pressures for cubic metals if physically-motivated embedding functions are used. Suprisingly, even if the angular character of the covalent bonding is included within TB description (1), the Cauchy pressure for most elemental and binary metallic systems remain positive due to the larger bond stress contribution. We have described the results of a detailed breakdown of the different contributions to the Cauchy pressure within the Harris-Foulkes approximation (HFA) to density functional theory. We show that negative values of the Cauchy pressure for both elemental transition metals such as Ir and binary intermetallics such as $\mathrm{Ti}_{3} \mathrm{Al}$, TiAl and $\mathrm{TiAl}_{3}$ are well reproduced by the HFA. More importantly, it is found that the electrostatic contribution including the double-counting part gives very large negative Cauchy pressure while the band energy contribution remains strongly positive. It gives us a background to conclude that the negative Cauchy pressure (NCP) arises namely from the environment dependence of the local TB orbitals which leads to the environmentally dependent overlap repulsion (NguyenManh et al 1998). The latter from the strong repulsion of valence $s p$ electrons as they squeeze into the ion core regions under the influence of the large covalent bonding forces.

We, therefore, represent this repulsion by a screened Yukawa-type potential (Nguyen-Manh et al 1998)

$$
U_{\mathrm{env}}=\frac{1}{2} \sum_{i, j \neq i} \frac{B}{R_{i j}} \exp \left(-\lambda_{i j}\left(R_{i j}-2 R_{\mathrm{c}}\right)\right)
$$

where $R_{\mathrm{c}}$ is the core radius and $\lambda_{i j}=(1 / 2)\left(\lambda_{i}+\lambda_{j}\right)$. The screening exponent, $\lambda_{i}$, is dependent on the local density or environment about atoms, as can be seen within a variationally-determined tight-binding (TB) approximation (Skinner and Pettifor 1991). By adding this environmentally-dependent contribution (7) to the binding energy (1), it is not difficult to find that the two Cauchy pressures can be determined by the following expressions

$$
C_{13}-C_{44}=C_{13}^{\text {bond }}-C_{44}^{\text {bond }}+C_{13}^{\mathrm{env}}-C_{44}^{\mathrm{env}}
$$




$$
\begin{aligned}
& +\frac{1}{4}\left(\sigma_{11}^{\text {bond }}+\sigma_{33}^{\text {bond }}+\sigma_{11}^{\text {env }}+\sigma_{33}^{\text {env }}\right) \\
& =C_{13}^{\text {bond }}-C_{44}^{\text {bond }}+\Delta_{C_{13}-C_{44}},
\end{aligned}
$$

and

$$
\begin{gathered}
C_{12}-C_{66}=C_{12}^{\text {bond }}-C_{66}^{\text {bond }}+C_{12}^{\text {env }}-C_{66}^{\text {env }}+\frac{1}{2}\left(\sigma_{11}^{\text {bond }}+\sigma_{11}^{\text {env }}\right) \\
=C_{12}^{\text {bond }}-C_{66}^{\text {bond }}+\Delta_{C_{12}-C_{66}}
\end{gathered}
$$

where $\sigma_{l v}$ are the appropriate stress components:

$$
\sigma_{\mu \nu}=\frac{1}{\Omega} \sum_{i, k>i} \frac{\partial U}{\partial R_{i k}^{\mu}} R_{i k}^{v},
$$

with $\Omega$ the volume of the system.

\subsection{Application for BOPs of TiAl}

A detailed account of using (1), (7)-(10) for constructing the BOPs for intermetallic alloys, TiAl, will be published elsewhere (Nguyen-Manh et al 2002a) and we briefly summarize the main results of our study. The attractive bond contribution is modelled within a simple $p-d$ tightbinding model which describes well the experimental trends within transition metal-metalloid binary compounds (Pettifor and Podloucky 1984). The model has been justified recently by a systematic LDAs study within density functional theory (Nguyen-Manh et al 1995; Nguyen-Manh and Pettifor 1999a, b) of structural trends within $\mathrm{AB}$ alloys. The bond integrals that enter the TB Hamiltonian are obtained directly from screened TBLMTO theory (Andersen and Jepsen 1984; Andersen et al 1985). Figure 2 shows the results for the $d d, p p$ and $d p$ bond integrals as a function of the bond length not only for $L 1_{0}-\mathrm{TiAl}$ but also for $D O_{19}-\mathrm{Ti}_{3} \mathrm{Al}$ and elemental $h c p$ $\mathrm{Ti}$ and $f c c$-Al (Znam et al 2000). We see that the bond integrals exhibit excellent transferability between different structures and compositions: $d d \boldsymbol{\sigma} d d \pi$ for Ti-Ti bonding ( $d d \boldsymbol{\delta}$ is not included in figure 2 but displays similar behaviour), $p p \sigma \quad p p \pi$ for $\mathrm{Al}-\mathrm{Al}$ bonding, and $p p \propto p p \pi$ for $\mathrm{Ti}-\mathrm{Al}$ bonding. A salient feature of the $\mathrm{Ti}-$ $\mathrm{Al}$ alloys, as well as of the pure $\mathrm{Ti}$ and $\mathrm{Al}$, is that their structures are close packed, so that their second nearest neighbour spacing is much larger than that of the first nearest neighbours.

The BOPs TiAl was constructed by fitting certain properties of the equilibrium $L 1_{0}$ structure. Hence, it is important to test the applicability of this potential to atomic configurations with environments very different from that encountered in the ground state $L 1_{0}$ lattice. Our first calculation tests the stability of the $L 1_{0}$ itself with respect to large homogeneous strain. This has been done by computing the building energy as a function of homogeneous expansions and contractions (by $\pm 20 \%$ ) of the two lattice parameters, $a$ and $c$. The resulting energy surface, which is shown in terms of the contour map in figure 3, possesses only one minimum, at the fitted equilibrium values, $a_{\exp }(=4.005 \AA)$ and $c_{\exp } \quad(=4.069 \AA)$. Hence, within the tested deformation range, the potential does not lead to any instabilities or unphysical metastable configurations. A comparison of the different contributions to the two Cauchy pressures ((8)-(9)) between the two BOP models for TiAl is presented in table 1. It shows clearly that the environmentally dependent term gives the negative contributions resulting in the correct sign of the Cauchy pressures in agreement with experiment. Here we use the BOPs with $c / a=1.0$ for investigating the energies of $\gamma \gamma$ interfaces in polysynthetically twinned (PST) single crystals (Yamaguchi and Inui 1993).

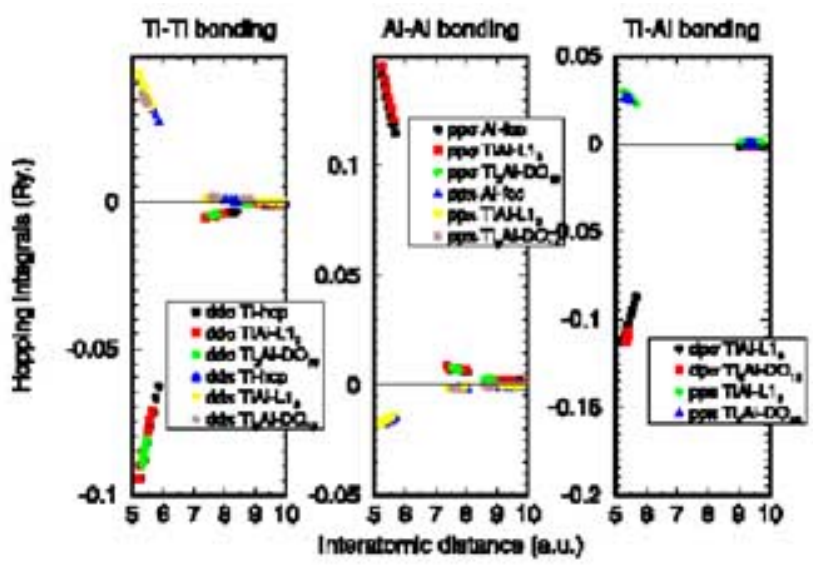

Figure 2. Bonding integrals in Ti-Al alloys calculated using TB-LMTO method as a function of interatomic distances.

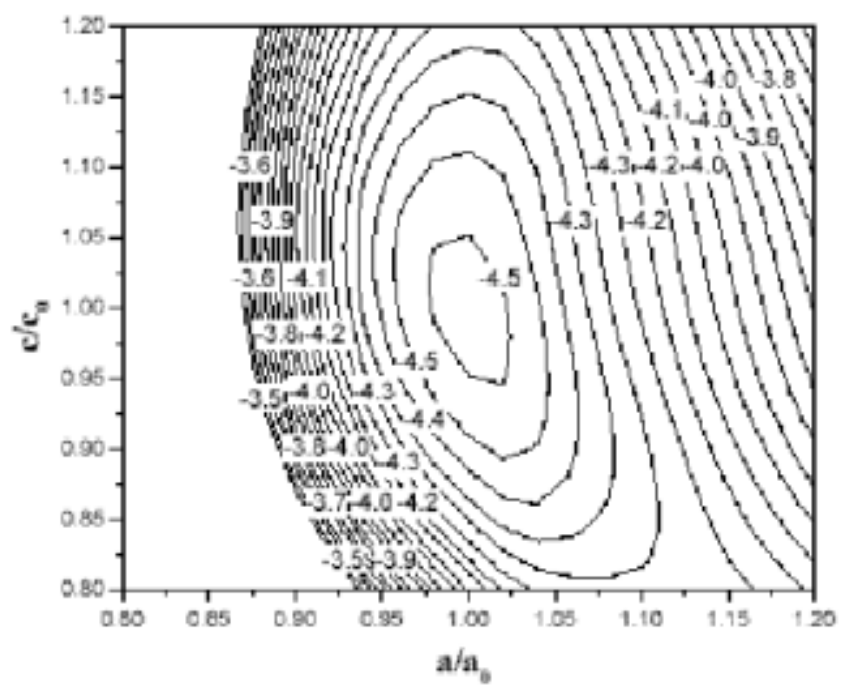

Figure 3. Energy surface (in meV/atom) for large homogeneous deformation of TiAl unit cell. 


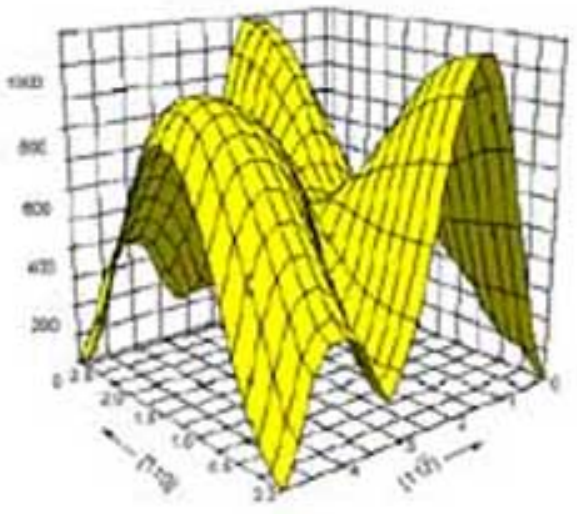

(a)

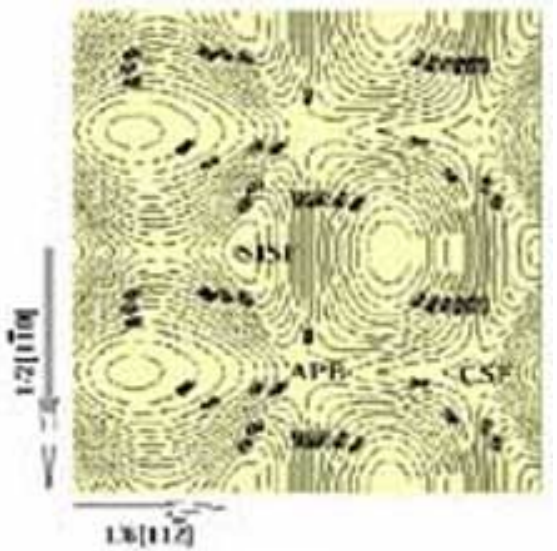

(b)

Figure 4. $\gamma$-surface for (111) plane in $\mathrm{mJ} / \mathrm{m}^{2}$ : (a) surface plot and (b) contour plot.
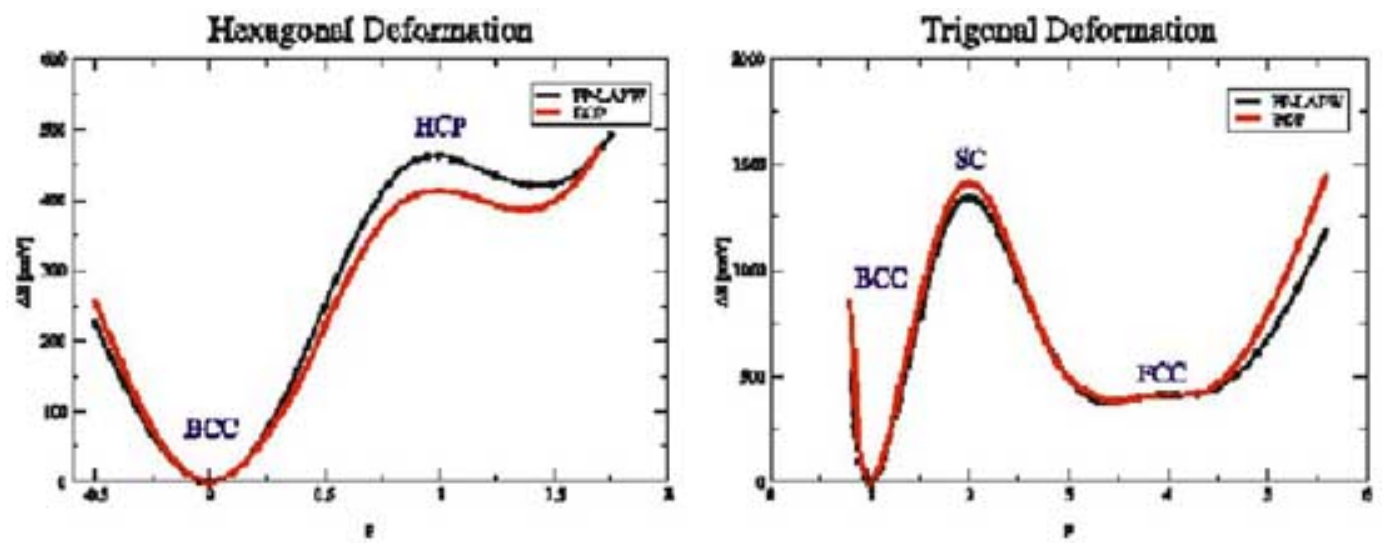

Figure 5. Deformation paths for $b c c$-Mo using environmentally dependent BOPs.

Table 1. Contributions (from (7)-(8)) to the Cauchy pressures (in $\mathrm{eV} / \AA^{3}$ ) for TiAl within two BOP models. Experimental values are taken from Tanaka et al (1996).

\begin{tabular}{lccc}
\hline $\begin{array}{l}\text { Cauchy } \\
\text { pressures }\end{array}$ & $\begin{array}{c}\text { BOP } \\
(c / a=1.016)\end{array}$ & $\begin{array}{c}\text { BOP } \\
(c / a=1.000)\end{array}$ & Experiment \\
\hline$C_{13}^{\text {bond }}-C_{44}^{\text {bond }}$ & 0.307 & 0.342 & - \\
$\Delta_{C_{13}-C_{44}}$ & -0.513 & -0.522 & - \\
$C_{13}-C_{44}$ & -0.206 & -0.180 & -0.213 \\
$C_{12}^{\text {bond }}-C_{66}^{\text {bond }}$ & 0.496 & 0.458 & - \\
$\Delta_{C_{12}-C_{66}}$ & -0.542 & -0.531 & - \\
$C_{12}-C_{66}$ & -0.046 & -0.073 & -0.040 \\
\hline
\end{tabular}

The close-packed $\{111\}$ planes play a key role in the plastic deformation of TiAl since they are the slip planes. We have, therefore, used the BOP to calculate the corresponding $\gamma$ surface (Vitek 1968). The predicted $\gamma$ surface is shown in figure 4 as a surface plot and as a contour plot. The three metastable stacking-fault-type defects on the (111) plane, which are seen in figure 5, are the antiphase boundary (APB) with the displacement (1/2) [101], complex stacking fault (CSF) with the displace- ment $(1 / 2) \quad[\overline{2} 11]$ and the super lattice intrinsic stacking fault (SISF) with the displacement (1/6) [11 $\overline{1}]$. The energies of these faults, which are predicted by BOP, are compared in table 2 with those evaluated by two different $a b$ initio methods: FLAPW (Ehmann and Fahnle 1998) and FP-LMTO (Vitek et al 1997) and also by a FinnisSinclair-type potential (Vitek et al 1997). The agreement between the BOP and $a b$ initio values are very good, in particular, reproducing correctly the relative high energy of the SISF. The Finnis-Sinclair-type potential, on the other hand, predicts much too low a value of this energy because the first and second nearest neighbour distances remain practically unchanged during the creation of this fault. The relatively large energy of the SISF results mainly from the change in the bond energy due to the angular dependence in the bond-order. In table 2, we also show our BOP prediction for the three different configurations of $\gamma \gamma$ interface (the $120^{\circ}$ rotational fault, the twin resulting from a $180^{\circ}$ rotation of the upper part of the crystal with respect to the lower part, and the pseudotwin which can be looked at as a combination of the $120^{\circ}$ rotational fault and the twin) and the twin boundary contain- 
Table 2. Stacking fault energies for (111) plane of TiAl calculated using the BOP with $c / a=1.016$. $\gamma / \gamma$ interfacial energies calculated using the BOP with $c / a=1 \cdot 0$. The BOP predictions $\left(\mathrm{in} \mathrm{mJ} / \mathrm{m}^{2}\right)$ are compared with two different $a b$ initio values and with Finnis-Sinclair (FS) calculations.

\begin{tabular}{lccccccc}
\hline- & APB & CSF & SISF & Twin & $120^{\circ}$ rot. & Pseudotwin & Twin with APB \\
\hline BOPs & 545 & 412 & 140 & $4 \cdot 2$ & 306 & 310 & 456 \\
FLAPW & 667 & 362 & 172 & 60 & 270 & 250 & 550 \\
FP-LMTO & 710 & 314 & 134 & 109 & 235 & 213 & 501 \\
FS & 275 & 275 & $2 \cdot 9$ & $1 \cdot 4$ & 144 & 145 & 288 \\
\hline
\end{tabular}

ing an additional APB shift with two different $a b$ initio calculations as well as those employing Finnis-Sinclair (FS) potentials. The agreement with the $a b$ initio is certainly very satisfactory. The case of the ordered twin again emphasizes the importance of directional bonding, as the low FS value has its roots, similarly as in the case of the SISF, in the fact that the first and second nearest neighbours distances are practically the same as in the ideal lattice.

\section{Environmentally dependent effect $I I$ : Screening of bonds}

\subsection{Discontinuities of bond integrals}

The environmental dependence of the TB parameters has been introduced in an ad-hoc fashion into numerous semi-empirical TB schemes. The Ames group (Tang et al 1996), in particular, have proposed writing the TB parameters in the form

$$
\tilde{\beta}_{l l^{\prime} \tau}^{i j}=\beta_{l l^{\prime} \tau}\left(\kappa R_{i j}\right)\left(1-S_{l l^{\prime} \tau}^{i j}\right),
$$

with $l, l^{\prime}=s, p$ or $d, \tau=\sigma \pi$ or $\delta$ and $A, \lambda$ and $\eta$ as fitting parameters. $\beta\left(\kappa R_{i j}\right)$ represents the two-centre bond integral between orbitals on sites $i$ and $j$ that have already been renormalized or contracted by the presence of their surrounding neighbours so that $\kappa$ is not a constant but is dependent on the local atomic density or effective coordination about the bond. The screening function, $S^{i j}$ represents the fact that the bonding between a given pair of atoms $i$ and $j$ is weakened by the presence of third atom $k$ in their vicinity. It accounts for the large discontinuity that is observed in the fitted $s s \boldsymbol{\sigma}$ bond integral curve between first and second nearest neighbours in elemental bcc molybdenum (Haas et al 1998). We have derived the analytic form of this screening function by starting from a non-orthogonal TB representation (Nguyen-Manh et al 2000). We have used BOPs theory (see \$2) to find the inverse non-orthogonality matrix, $(I+O)^{-1}$, by analogy with our previous derivation of the Green's function matrix, $(\boldsymbol{\varepsilon}-H)^{-1}$. Setting $\varepsilon=1$ and $H=-O$, where $I$ and $O$ are the unit and overlap matrices, respectively, gives analytic expressions for the screening functions for $\sigma \pi$ and Sbonds

$$
S_{l l^{\prime} \tau}^{i j}=\frac{\left(c_{1}^{i j}\right)_{l l^{\prime} \tau}-\left(\overline{\boldsymbol{\mu}}_{2}\right)_{l l^{\prime} \tau}+\left(\overline{\boldsymbol{\mu}}_{3 l^{\prime} \tau}\right)}{1+O_{l l^{\prime} \tau}^{2}\left(R_{i j}\right)-2\left(\overline{\boldsymbol{\mu}}_{2}\right)_{l l^{\prime} \tau}+\left(\overline{\boldsymbol{\mu}}_{3}\right)_{l l^{\prime} \tau}} .
$$

We find that the main contribution to $S^{i j}$ is coming from $c_{1}{ }^{i j}$ which is the bond-overlap interference contribution linking orbitals $|i \mu \nu \tau\rangle$ and $\left|j v l^{\prime} \tau\right\rangle$, viz.

$$
\begin{aligned}
& \left(c_{1}^{i j}\right)_{l l^{\prime} \tau}=\sum_{k \neq i, j}\left[\left(1+\delta_{\tau 0}\right) / 4\right]\left\{\left[\beta_{l s \sigma}\left(R_{i k}\right) O_{s l^{\prime} \sigma}\left(R_{k j}\right)\right.\right. \\
& \left.+O_{l s \sigma}\left(R_{i k}\right) \beta_{s l^{\prime} \sigma}\left(R_{k j}\right)\right] g_{l \tau}\left(\theta_{j i k}\right) g_{l^{\prime} \tau}\left(-\theta_{i j k}\right) \\
& +\left[\beta_{l s \sigma}\left(R_{i k}\right) O_{s l \sigma}\left(R_{k i}\right) O_{l l^{\prime} \tau}\left(R_{i j}\right) g_{l \tau}^{2}\left(\boldsymbol{\Theta}_{j i k}\right)\right. \\
& \left.\left.+O_{l l^{\prime} \tau}\left(R_{i j}\right) O_{l^{\prime} s \sigma}\left(R_{j k}\right) \beta_{s l^{\prime} \sigma}\left(R_{k j}\right) g_{l^{\prime} \tau}^{2}\left(\Theta_{i j k}\right)\right]\right\} / \beta_{l l^{\prime} \tau}\left(R_{i j}\right),
\end{aligned}
$$

with angular functions defined by $g_{0 \sigma}(\boldsymbol{\theta})=1 ; \quad g_{1 \sigma}(\boldsymbol{\theta})=$ $\cos \boldsymbol{\theta}, \quad g_{1 \pi}(\boldsymbol{\theta})=\sin \boldsymbol{\theta}, \quad g_{2 \sigma}(\boldsymbol{\theta})=(1 / 4)(1+3 \cos 2 \boldsymbol{\theta}), \quad g_{2 \pi}(\boldsymbol{\theta})=$ $(\sqrt{3} / 2) \sin 2 \boldsymbol{\theta}, g_{2 \delta}(\boldsymbol{\theta})=(\sqrt{3} / 4)(1-\cos 4 \boldsymbol{\theta})$.

\subsection{Application for BOPs in bcc-like materials}

The power of this novel analytic screening function will be demonstrated by considering the environmental dependence of the bond integrals in elemental $b c c$ Mo, model $b c c \mathrm{Si}$ and the binary $b c c$-related $C 11_{b} \mathrm{MoSi}_{2}$ (NguyenManh et al 2000). We find that there is a large discontinuity between the first and second nearest neighbours for the $p p \propto p p \pi$ and $d d \pi$ bond integrals, but that this discontinuity is absent for $d d \sigma$ There is a noticeable environmental dependence in the behaviour of the first nearest neighbour $p p \pi$ bond integral in going from elemental bcc Si to binary $C 11_{b}-\mathrm{MoSi}_{2}$. This behaviour can be traced directly to the angular dependence of the screening function which is absent in the empirical form of (11). The $b c c$ lattice (and the closely related $C 11_{b}$ lattice) have nearest neighbour bond angles of $\cos ^{-1}(1 / \sqrt{3})$ so that $g_{2 \sigma}=(1 / 4)(1+3 \cos 2 \theta)$ vanishes identically, causing the surrounding neighbours to leave the $d d \sigma$ bond unscreened. On the other hand, the second nearest neighbour $d d \pi$ bond is heavily screened, both its magnitude and slope being reduced by a factor of 3 . This is 
critical for the behaviour of the second nearest neighbour force constants and removes the problem of the unstable $T 2$ phonon mode at the $N$ point that is found in most twocentre TB fits. For the case of $b c c-\mathrm{Mo}$, using unscreened bonding integrals we find that $\omega_{\mathrm{r}}(T 2)=1.0 \mathrm{THz}, \omega_{\mathrm{N}}$ $(T 1)=6.3 \mathrm{THz}, \quad \omega_{\mathrm{N}}(L)=5.2 \mathrm{THz}, \quad$ whereas within the present formalism the corresponding values of $3.6,5.9$ and $8.3 \mathrm{THz}$ are consistent with the experimental values of $4 \cdot 5,5 \cdot 8$ and $8 \cdot 1 \mathrm{THz}$, respectively.

We have used the analytic screening function, $S^{i j}$, to construct the BOPs for $b c c$ transition metals (Mo, Nb, Ta, W), from the binding energy defined by (1) and (7) (Mrovec et al 2001). Since the potentials are intended for atomistic modelling of extended defects they have to be applicable for distorted structures along certain deformation path (Paidar et al 1999; Nguyen-Manh et al 1996; Nguyen-Manh and Pettifor 1999b). Figure 5 shows the dependence of the energy per atom, relative to the energy of the $b c c-\mathrm{Mo}$, on the deformation parameter, $p$, for deformation path between the $b c c$ and $h c p$ lattices (hexagonal deformation) and between the $b c c$ and $f c c$ lattices passing through the simple cubic (sc) lattice (trigonal deformation). The results demonstrate an excellent agreement between BOPs and $a b$ initio (FLAPW) predictions; in particular the auxiliary minima found in the vinicity of $p=4$ for the trigonal path depend strongly on the interatomic forces.

As a prelude to dislocation simulations, we have investigated the $\gamma$ surface for the $\{110\}$ split plane which provides some insight into the shape and extension of the dislocation core. Quantitatively, its shape is similar to that obtained from the unscreened BOPs but the maximum energy is about $40 \%$ higher and thus the surface is generally steeper. This suggests a narrow core since spreading of dislocation into $\{110\}$ plane becomes energetically less favourable. The differential displacement map (Vitek 1992) of the core structure calculated with the environmentally dependent BOPs is shown in figure 6 . This core displays three-fold symmetry but is much narrower and closer to the six-fold symmetry than the core found in studies employing central forces (FinnisSinclair) potentials. More importantly, this structure is close to that found in recent $a b$ initio calculations using Green's function boundary conditions (Rao and Woodward 2001).

\section{Anisotropy of interactions}

Another important application of the analytic expression for the screening function (12) of the bond integrals is that it enables us to explain the origin of anisotropic bonding in layered materials. Graphite is a prototypical one characterized by exceptionally strong $s p^{2}$ covalent interlayer bonding and weak interlayer bonding. The highly anisotropic bonding gives rise to a number of unusual properties that are of long-standing technological and scientific importance such as the graphite intercalation compounds or outstanding chemical and physical properties of amorphous carbon (Mckenzie 1996). Recent attempts to simulate the thin-film depositions of amorphous carbon using empirical potentials (Tersoff, Brenner, Stillinger-Weber) have not been successful because they are transferable poorly in different environment due to short-ranged interactions. Our systematic study of band energy in graphite using the third-generation LMTO method demonstrates clearly that interlayer hopping integrals play an important role in understanding of its electronic structure (Nguyen-Manh et al 2002b). Here we find by using our formalism of screening for $\sigma$ and $\pi$ bonds (within the $s p^{3}$ basis) that the interlayer bond integrals corresponding to fourth and fifth nearest neighbours (NN) are almost unscreened by their environment while the second and third $\mathrm{NN}$ bond integrals are strongly screened as it is shown in figure 7. More crucially, figure 7 predicts an excellent transferability of TB bond integrals between graphite and diamond structures leading to the construction of transferable BOPs for carbon beyond $1 N N$ approximation which has been used in previous studies (see for example, Pettifor and Oleinik 2000).
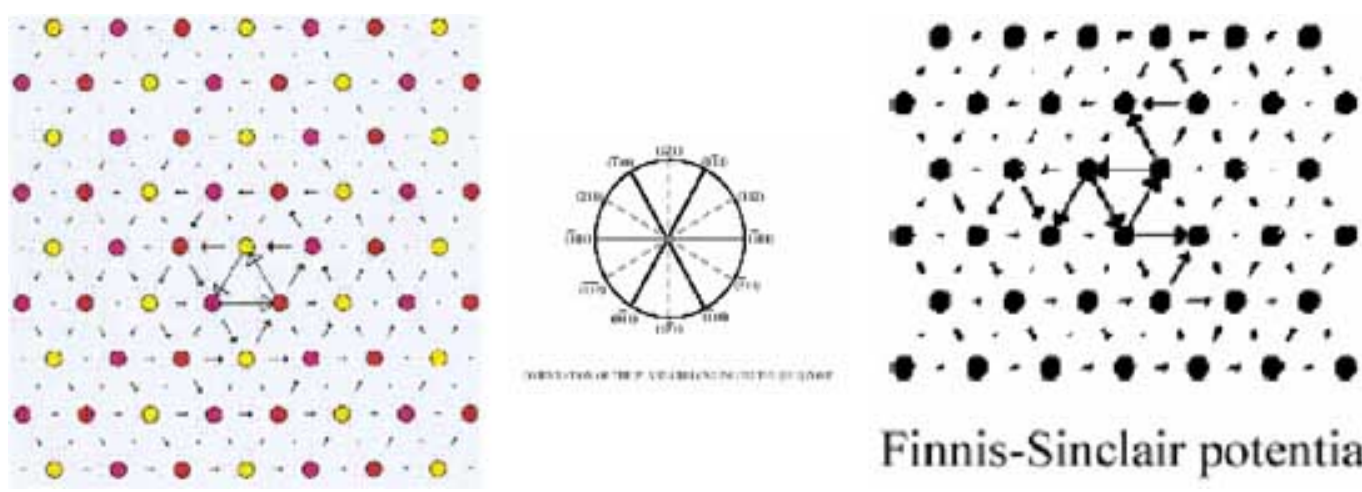

Finnis-Sinclair potential

Figure 6. Core structure of the (1/2) $\rangle 111<$ (screw component) for molybdenum. 
GRAPHITE
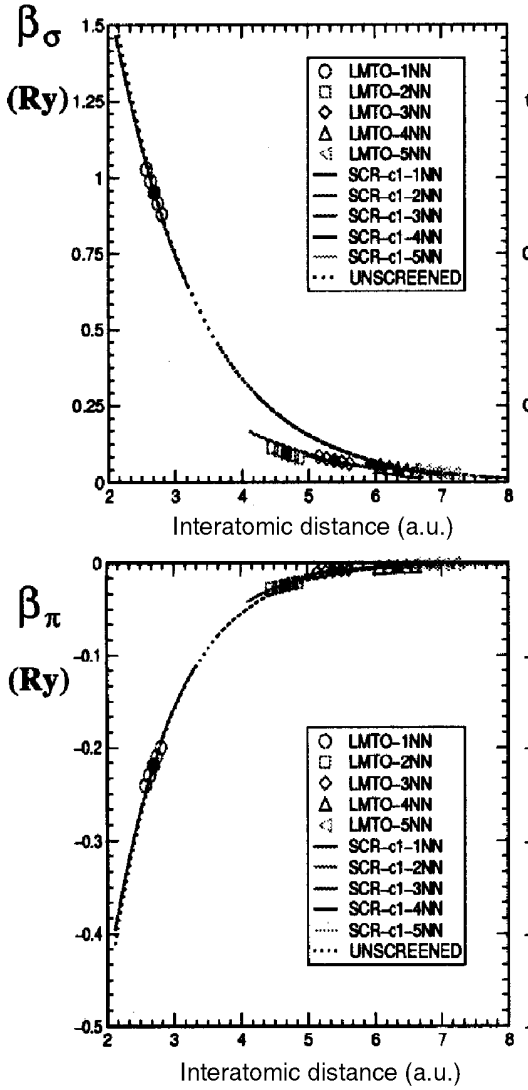

DIAMOND

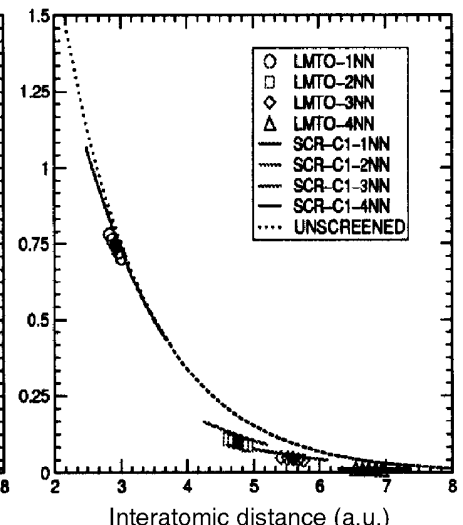

Interatomic distance (a.u.)

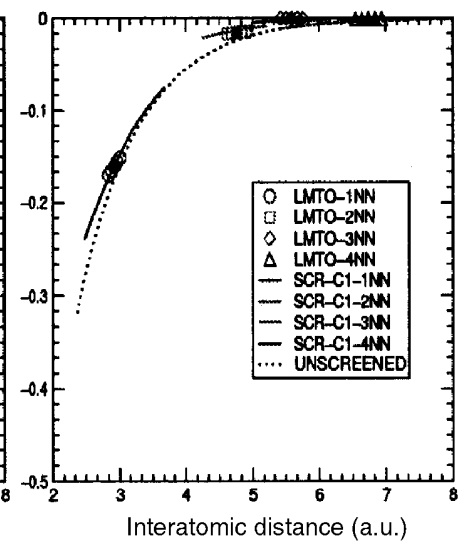

Figure 7. Transferability of $\sigma$ and $\pi$ bond integrals between graphite and diamond structure for carbon.

\section{Conclusions}

We have demonstrated that the environmental dependence affects strongly both repulsive and bonding contributions within two-centre orthogonal TB scheme. Our developed physics concepts of screening atom and screening bond integrals which result in their environmental dependence substantially improve the transferability of constructed BOPs. This provides the reliable method for predicting the analytic behaviour of transferable TB parameters and help extend the accuracy and applicability of BOPs to technologically important areas such as the CVD growth of diamond films or mechanical properties of high-temperature intermetallics.

\section{Acknowledgements}

This work is supported by the U.S. Department of Energy, BES Grant No. DE-PG02-98ER45702 and by the UK-EPSRC Grant. (DNM) would like to acknowledge stimulating discussions with $\mathrm{OK}$ Andersen, $\mathrm{M}$ Aoki, D Dimiduk, M Fahnle, YW Kim, V Paidar, K Tanaka, C Woodward and M Yamaguchi.

\section{References}

Aoki M 1993 Phys. Rev. Lett. 713842

Andersen O K and Jepsen O 1984 Phys. Rev. Lett. 532571

Andersen O K, Jepsen O and Glotzel D 1985 in Highlights of condensed matter theory (eds) F Bassani et al (Amsterdam: North-Holland)

Andersen O K, Arcangeli C, Tank R W, Saha-Dasgupta T, Krier G, Jepsen O and Dasgupta I 1998 in Tight-binding approach to computational materials science, MRS Proceedings (eds) $\mathrm{P}$ E A Turchi et al (Warrendale: Materials Research Society) 491 p. 3

Coulson C A 1939 Philos. Trans. R. Soc. London A169 413

Daw M S and Baskes M I 1984 Phys. Rev. B29 6443

Dimiduk D M 1999 Mater. Sci. Eng. A263 281

Ehmann J and Fahnle M 1998 Philos. Mag. A77 701

Finnis M W and Sinclair J E 1984 Philos. Mag. A50 45

Haas H, Wang C Z, Fahnle M, Elsaaer C and Ho K M 1998 Phys. Rev. B57 1461

Mrovec M, Nguyen-Manh D, Pettifor D G and Vitek V 2001 MRS Symp. Proc. 653 Z6.3

Mckenzie D R 1996 Rep. Prog. Phys. 593611

Nguyen-Manh D and Pettifor D G 1999a Intermetallics 71005

Nguyen-Manh D and Pettifor D G 1999b in Gamma titanium aluminides (eds) Y W Kim et al (Pittsburgh: TMS Publications) p. 175 
Nguyen-Manh D, Bratkovsky A M and Pettifor D G 1995 Philos. Trans. R. Soc. London A351 529

Nguyen-Manh D, Pettifor D G, Shao G, Miodownik A P and Pasturel A 1996 Philos. Mag. A74 1385

Nguyen-Manh D, Pettifor D G, Znam S and Vitek V 1998 in Tight-binding approach to computational materials science, MRS Proceedings (eds) P E A Turchi et al (Pittsburgh: Materials Research Society) 491 p. 353

Nguyen-Manh D, Pettifor D G and Vitek V 2000 Phys. Rev. Lett. 854136

Nguyen-Manh D, Znam S, Pettifor D G and Vitek V 2002a, to be published

Nguyen-Manh D, Saha-Dasgupta T and Andersen O K 2002 Bull. Mater. Sci. 2627

Paidar V, Wang L G, Sob M and Vitek V 1999 Model. \& Simul. Mater. Sci. Eng. 7369

Pettifor D G 1997 Phys. Edu. 32164

Pettifor D G and Podloucky R 1984 Phys. Rev. Lett. 531080

Pettifor D G and Oleinik I I 2000 Phys. Rev. Lett. 844124

Pettifor D G, Aoki M, Gumbsch P, Horsfield A P, NguyenManh D and Vitek V 1995 Mater. Sci. Eng. A192-193 24

Rao S I and Woodward C 2001 Philos. Mag. A81 1317
Skinner A J and Pettifor D G 1991 J. Phys. Condens. Matter 3 2029

Sutton A, Finnis M W, Pettifor D G and Ohta Y 1988 J. Phys. C21 35

Tanaka K, Ichitsubo T, Inui $\mathrm{H}$, Yamaguchi $\mathrm{M}$ and Koiwa $\mathrm{M}$ 1996 Philos. Mag. Lett. 7371

Tang M S, Wang C Z, Chan C T and Ho K M 1996 Phys. Rev. B53 979

Tersoff J 1986 Phys. Rev. Lett. 56632

Vasudevan A K, Petrovic J J, Fishman S G and Nathal M V (eds) 1999 Mater. Sci. Eng. A261 1

Vitek V 1968 Philos. Mag. A18 773

Vitek V 1992 Prog. Mater. Sci. 361

Vitek V, Ito K, Siegl R and Znam S 1997 Mater. Sci. Eng. A240 752

Yamaguchi M and Inui H 1993 in Structural intermetallics, Proc. of international symposium on structural intermetallics (eds) R Darolia et al (Warrendale, PA: TMS Publications) p. 27

Znam S, Nguyen-Manh D, Pettifor D G and Vitek V 2000 in Properties of complex inorganic solids (eds) A Meike et al (Dordrecht, The Netherlands: Kluwer Academic/Plenum) Vol. 2, p. 479 